\title{
Evaluating Variable Speed Limits and Dynamic Lane Merging Systems in Work Zones: A Simulation Study
}

\author{
Zaier Zaidi, ${ }^{1}$ Essam Radwan, ${ }^{2}$ and Rami Harb ${ }^{2}$ \\ ${ }^{1}$ Planning Wing, National Highway Authority, Islamabad 44000, Pakistan \\ ${ }^{2}$ CATSS, Department of Civil and Environmental Engineering, University of Central Florida, Orlando, FL 32816, USA
}

Correspondence should be addressed to Zaier Zaidi, zaierabbas@yahoo.com

Received 29 February 2012; Accepted 1 May 2012

Academic Editors: P. J. S. Cruz and I. Raftoyiannis

Copyright ( 2012 Zaier Zaidi et al. This is an open access article distributed under the Creative Commons Attribution License, which permits unrestricted use, distribution, and reproduction in any medium, provided the original work is properly cited.

\begin{abstract}
ITS technologies such as dynamic lane merging (DLM) and variable speed limits (VSL) have been deployed in roadway work zones in an attempt to enhance safety and mobility through vehicular traffic. The DLM system in its two main forms namely the early merge and the late merge was designed to advise drivers on definite merging locations whereas VSL were introduced to work zones to decrease speed fluctuations, variances, and to smoothen traffic through work zones. Up to date, there are no studies that contrast Maintenance Of Traffic (MOTs) plans including a combination of ITS technologies to standard work zones MOTs under matching work zone settings. This study simulates a two-to-one work zone lane closure configuration in VISSIM under six different MOT plans and compares work zone throughputs and travel times across MOTs namely Motorist Awareness System (MAS), early DLM, late DLM, VSL combined with MAS, a VSL and early DLM combination, and a VSL late DLM combination. Results showed that the combination of VSL and DLM or standalone DLM improve work zone throughputs and travel times compared to work zones with conventional static Florida MOT and work zones with VSL.
\end{abstract}

\section{Introduction}

To improve traffic safety and mobility in work zone areas, several states of the USA explored the DLM and VSL systems. The DLM systems are intelligent work zone traffic control systems that respond to real-time traffic changes via traffic sensors. The DLM systems are designed to advise drivers on definite merging locations and can take two forms; dynamic early merge and dynamic late merge. The idea behind the dynamic early merge is to create a dynamic nonpassing zone, to encourage drivers to merge into the open lane before reaching the end of a queue and to prohibit them from using the closed lane to pass vehicles in the queue and merge into the open lane ahead of them [1]. The concept behind late merge is to make more efficient use of roadway storage space by allowing drivers to use all available traffic lanes to the merge point. Once the merge point is reached, the drivers in each lane take turns proceeding through the work zone [2].

Several studies were undertaken to contrast the early form of the DLM $[1,3-5]$ or the late form of the DLM $[2,6-11]$ to existing maintenance of traffic (MOT) plans or standard MUTCD work zone traffic control plans. Whereas each study exposes the advantages and disadvantages of the tested systems, up to date there are no studies that cross compare both merging schemes (i.e., early and late DLM) under the same work zone settings.

VSL systems are a type of intelligent transportation systems technology that involves the setting of maximum and or minimum speed limits. VSLs display speed limits based on observed real time traffic conditions and or weather conditions. Several studies [12-14] showed the advantages of implementing VSLs in work zones and the resulting positive effects in reducing speed variance and speed fluctuation under certain conditions. However, there is a lack of literature in the examination of VSL and DLM systems' combinations in work zones.

This study incorporates the early and late forms of DLM system and the VSL system into the Florida conventional MOT plans known as the Motorist Awareness System (MAS) [15]. Six resulting modified MOT plans for a two-to-one work zone lane closure configuration were simulated. The first MOT consisted of the conventional plans used in Florida 
work zones known as the Motorist Awareness Systems (MASs). The 5 remaining MOTs consisted of supplementing the MAS plans with one or a combination of DLM and VSL systems. Therefore, the second MOT (or modified MAS) consisted of a Dynamic early Merging System (early DLM), the third MOT consisted of a Dynamic late Merging System (late DLM), the fourth MOT consisted of appending a VSL to the conventional Florida MAS, the fifth MOT consisted of adding a VSL to the early DLM, and the sixth MOT consisted of adding a VSL to the late DLM. The operational effectiveness of each MOT type was determined for different levels of drivers' adherence to the messages displayed by the systems or compliance rate, different levels of truck percentages in the traffic composition, and different levels of demand volumes. Details about these MOT plans are presented in the following sections.

There exist a wide range of tools to evaluate the safety and mobility of drivers at work zone lane closures. QUEWZ, QuickZone, and DELAY Enhanced 1.2 are analytical tools developed to assess traffic impacts at work zones. However, these tools in addition to the methodology presented by HCM 2000 do not offer the flexibility of adjusting for the lane management strategy (DLM) and assigning variable speeds as suggested in this study. VISSIM is microscopic stochastic simulation package that enables creating specific scenarios (e.g., DLM and VSL logic) via vehicle actuated programming (VAP). A program reflecting our algorithm (DLM and VSL logic) was coded to communicate with VISSIM in realtime. The next sections introduce VISSIM and elaborate on the methodology followed in simulating the dynamic lane merging in VISSIM.

The main objective of this research is to compare operations amongst combinations of VLS and dynamic lane merging and the conventional MAS used in Florida. Work zone throughputs and travel times are the selected measures of effectiveness and are compared across the six different MOT types. Results and recommendations on the simulation of the tested systems are presented. It should be noted that the scope of the objective is restricted to freeway work zones consisting of a two-to-one lane closure configuration.

The remainder of this paper is organized as follows. Brief description of all the six scenarios, namely, (work zone without VSL and without DLM, work zone with VSL and without DLM, work zone with VSL and early DLM, work zone with VSL and late DLM, work zone with early DLM and without VSL, and work zone without VSL and with late DLM), VISSIM simulation including network coding and VAP coding to mimic both VSL and DLM logic. Finally, scenario runs with different levels of drivers' compliance, truck percentages, and demand volumes are presented, followed by conclusions and recommendations.

\section{Scenario Description}

2.1. Motorist Awareness System (MAS). Currently the Florida Department of Transportation deploys an MOT plan known as the MAS. According to the Florida Plans Preparation Manual (PPM), the MAS consists of Portable Regulatory
Signs (PRSs) highlighting the regulatory speed for the work zone and a Radar Speed Display Unit (RSDU) displaying the motorist's work zone speed. The MAS also comprises a Portable Changeable Message Sign (PCMS), a lane drop warning sign, a speeding fines doubled warning sign, in addition to road work ahead warning signs [15]. Figure 1 shows the MAS plans.

2.2. Early/Late DLM and VSL. The early and late DLMs consist of supplementing the conventional MAS plans with one Portable Changeable Message Sign (PCMS) and a nonintrusive sensor (Remote Traffic Microwave Sensor, RTMS) trailer as shown in Figure 1. The messages displayed by the PCMS define whether the system is an early or late DLM system. To include the VSL in the MOT plans, The PRS (used in MAS) is replaced by a PCMS that displayed the average speed over every 2-minute time interval. It should be noted here that the location of the both the additional PCMSs and the sensor trailer are identical for all VSL, early and late DLM combinations.

The DLM operates under two modes; the passive mode and the active mode. Under the passive mode the additional PCMS is set to display a flashing "CAUTION/CAUTION" message for both the early and late DLM. Under the active mode, the PCMS displays "DO NOT PASS" followed by "MERGE HERE" alternately for the early DLM and "STAY IN YOUR LANE' followed by “MERGE AHEAD” alternately for the late DLM. The early and late DLMs are activated once the average speed over any 2-minute time interval drops below $50 \mathrm{mph}$. The DLM will be deactivated (passive mode) once the average speed over the next time stamp goes over $50 \mathrm{mph}$. It should also be noted that the minimum activation time of the PCMS was set for 5 minutes. Similarly, the speed of vehicles at advance warning area is calculated during a cycle time of 2-minutes and the corresponding average speed distribution is posted at the VSL in the increments of $5 \mathrm{mph}$. If the average speed drop is more than $5 \mathrm{mph}$, the VSL will display the reduced speed, that is, 5 miles less than the previously posted speed until the average speed goes beyond the posted speed.

\section{Vissim Simulation}

VISSIM is a microscopic, time step, and behavior-based simulation model. VISSIM is a commercially available traffic simulation package developed by PTV AG, Karlsruhe, Germany and distributed in the United States by PTV America, Inc. [16].

The work zone with a two-to-one lane closure configuration was coded in VISSIM through a series of links, connectors, routing decisions, and lane closures to represent the actual geometry of the work zone. Figure 2 shows a sketch of the modified MOT plans for the 2-to-1 lane closure and the corresponding resulting nodes and roadway segments in VISSIM. Figure 2 shows 6 links and 5 nodes. The first node represents the first work zone PCMS. The second node represents the location of the VSL, whereas node 3 shows the location of additional PCMS where merging information is 


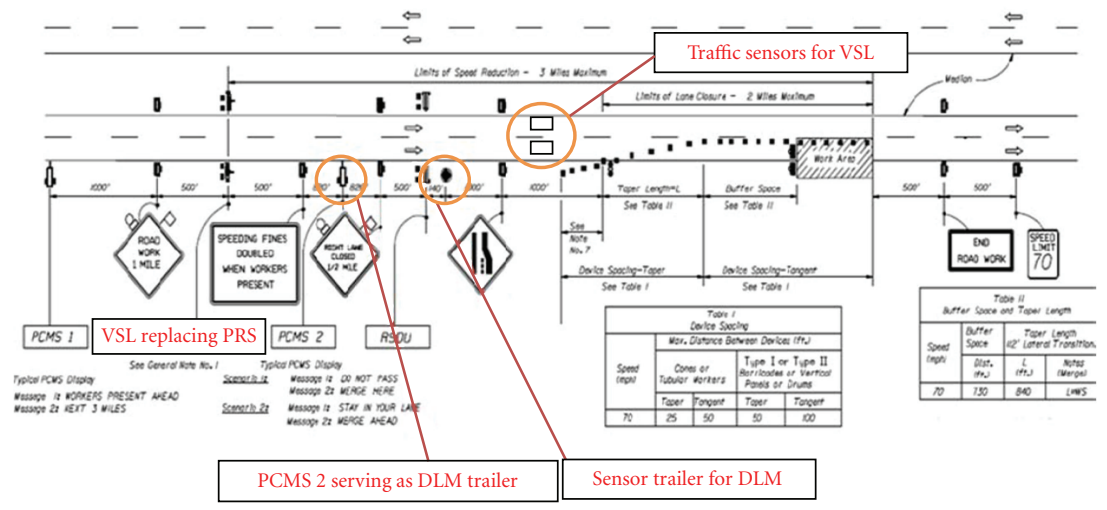

FIgURE 1: MAS plans and modification.

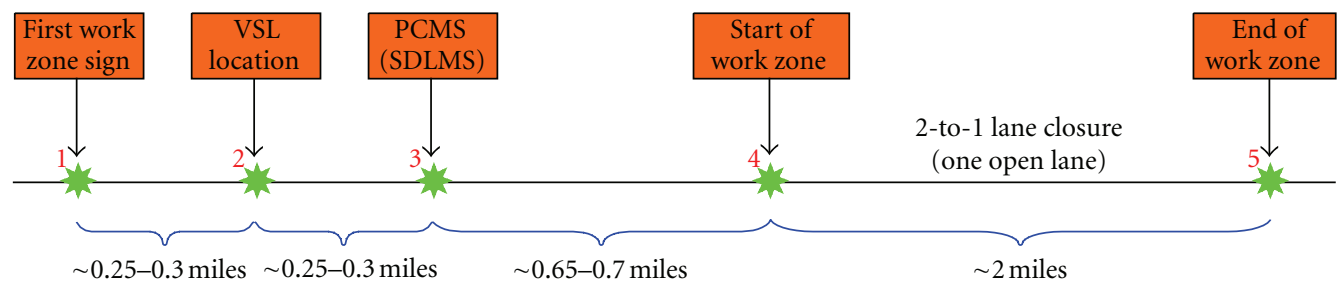

FIGURE 2: Modified MOT plan replication in VISSIM.

provided to drivers. Node 4 represents the lane closure start (one open lane). Node 5 represents the lane closure end (two lanes open).

The next step was to mimic the VSL and DLM logic in VISSIM. For the DLM logic, the Remote Traffic Microwave Sensor (RTMS) captures the average speed of vehicles over two-minute time intervals and the built-in algorithm checks if the speed threshold is reached. If the speed threshold is reached, the additional Portable Changeable Message Sign (PCMS) displays the necessary messages. The PCMS keeps displaying the messages until another speed threshold is reached. When the early DLM message is displayed, drivers merge to the open lane. When the late DLM message is displayed, drivers stay in their lane until the taper. Similarly, the speed of vehicles at advance warning area is calculated during a cycle time of 2 minutes and the corresponding speed limit is posted on the VSL in the increments of $5 \mathrm{mph}$. If the average speed drop is more than $5 \mathrm{mph}$, the VSL will display the reduced speed, that is, $5 \mathrm{mph}$ lower than the previously posted speed until the average speed goes beyond the posted speed.

To imitate the early DLM in VISSIM, partial decision routings were designated. Drivers either follow a decision routing designated to merge early (when speed drops below threshold) or follow a random merging (when speed remains above speed threshold). For instance in Figure 3 the striped region is designated as partial route 1 (PCMS activated, early merge instructions) and the solid color region is designated as partial route 2 (PCMS not activated, random merging). The alternation between partial route 1 (early merge/PCMS activated) and partial route 2 (PCMS not activated, random merging) was controlled by the VAP. Two loop detectors were placed (in VISSIM) at the same location of the RTMS. The loop detectors in VISSIM capture individual vehicles speed. These loop detectors can communicate with signal controllers and can only interact with traffic signals. Since loop detectors cannot directly communicate with the routing decision, Vehicle Actuated Programming (VAP) is used. VAP "is an optional add-on to VISSIM for the simulation of programmable, phase or stage based, traffic actuated signal controls. The control logic is coded in a txt file format and the VAP interprets the control logic commands and creates commands for the VISSIM network. At the same time various detectors variables reflecting the current traffic situation are retrieved from the simulation and processed in the logic" [16]. The same logic was followed for coding the late DLM in VISSIM. However, three partial routes were created. Partial routes 2 and 3 for late merge and partial route 1 for random merging (see Figure 3). As for the MAS one static routing decision was created.

For the VSL algorithm, two loop detectors were introduced in the advance warning area (in the VISSIM model) to calculate the average speed for the VSL. Another VAP was coded to dynamically modify the speeds of vehicles. Two desired speed decision points are placed in each lane 4,780 feet ( 0.9 miles) upstream of the taper. These desired speed decision points served as VSL with their posted speed changes in accordance with the VAP logic. These desired speed decision points are placed at the same location where PRS sign was placed. Figure 3 illustrates VSL desired speed decision points for both early and late DLM+VSL combinations. 


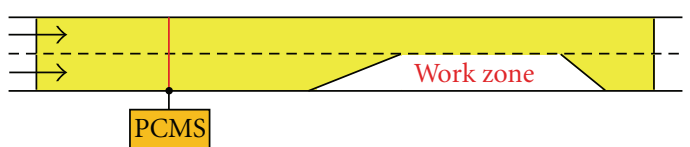

$\square$ Static route

(a)

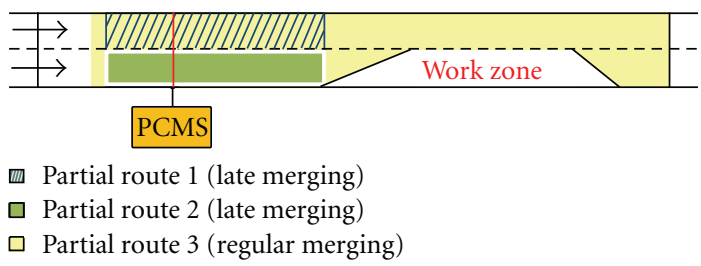

(c)

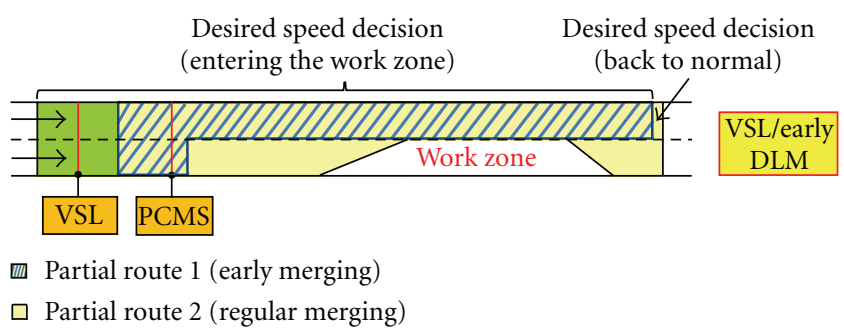

(e)

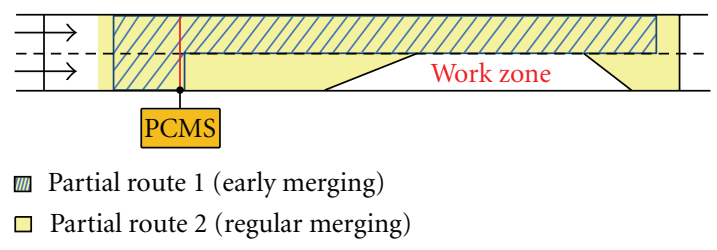

(b)

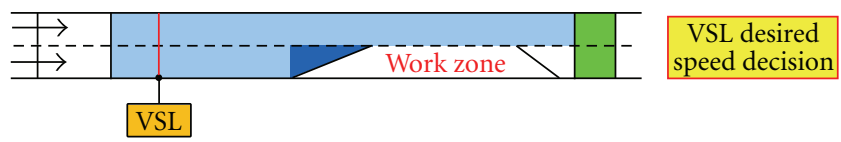

$\square$ Desired speed decision (entering)

$\checkmark$ Desired speed decision (back to normal)

(d)

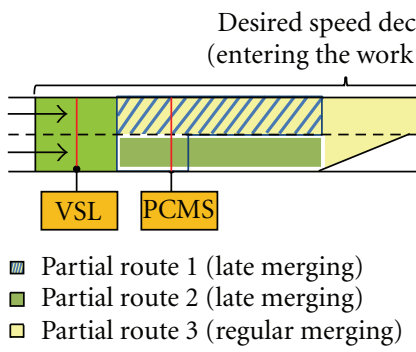

(f)

FIgURE 3: Decision routings and desired Speed decision.

An important factor in the DLM effectiveness is the driver's compliance rate to the messages displayed by the PCMS. To reflect compliance rate in the simulation model, and since partial routing decision can control specific vehicle classes, three vehicle classes are created; obey car, obey truck, disobey car and disobey truck. obey car, and obey truck vehicle classes represent the vehicles that are controlled by the partial routing decision therefore complying with the PCMS messages. The disobey car and disobey truck are not controlled by partial routing decision constituting the noncomplying vehicles.

\section{Field Data Collection and Vissim Calibration}

Field data was collected on Interstate-95 in Malabar, Florida. I-95 is 2-lane per direction limited access rural freeway with $70 \mathrm{mph}$ speed limit (reduced to $60 \mathrm{mph}$ during work). The work zone consisted of a resurfacing and milling job on the south bound of I-95 on a 13 mile stretch. A 2 to 1 lane closure configuration was adopted and the work zone moved on a daily basis covering a length of approximately 3 miles per day. Data was collected on homogenous basic freeway segment of I-95 with no on/off ramps. For more information about the field study, readers may refer to Harb et al., 2011 [17].

The calibration process in VISSIM was divided into several steps. First, travel time through the work zone was selected as the index of comparison. Second, the required number of simulation runs was determined. Third, an initial evaluation was conducted with the VISSIM's driving behavior's default parameters. Fourth, an examination of the key parameters was conducted and calibration parameters were determined. Multiple runs with different values of the key parameters were run by trial, and error until the calibration is completed. Fifth, for the model validation, the work zone throughput (different dataset) was used to verify the homogeneity between the real and simulated environment. Readers may refer to Harb et al., 2010 [15] for more information about the simulation model calibration and validation.

\section{Simulation and Results}

As mentioned earlier, the objective of the simulation study is to determine the effectiveness of each MOT type (early DLM, late DLM, early/late combinations with VSL, VSL alone and MAS) under different driver's compliance rate, different truck percentage in the traffic composition, and different traffic demand volumes. For that purpose, different levels of each of these variables were considered. Three different levels of drivers' compliance rate, C20 (20\% of drivers comply with the merging instruction), C40 ( $40 \%$ of drivers comply with the merging instruction), and C80 (80\% of drivers comply to the merging instruction) are created. Three different levels of truck percentage in the traffic composition are created, T10 (trucks constitute $10 \%$ of the demand volume), T20 (trucks constitute $20 \%$ of the demand volume), and T30 (trucks constitute $30 \%$ of the demand volume). Five different 


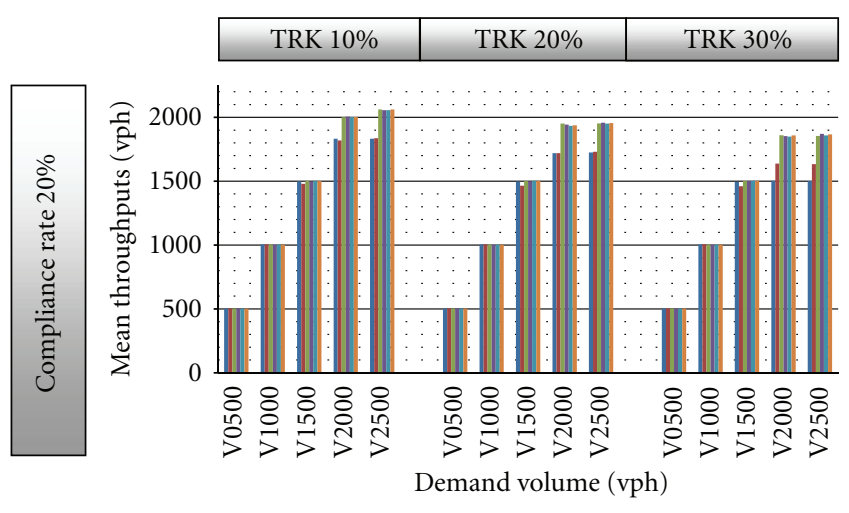

(a)
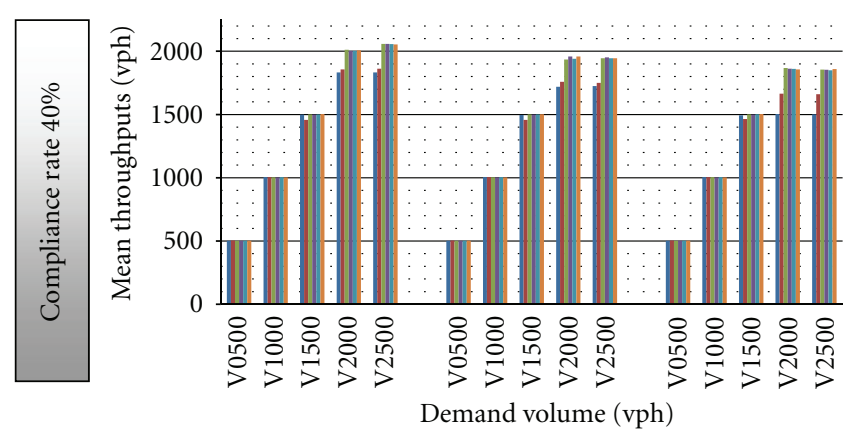

(b)

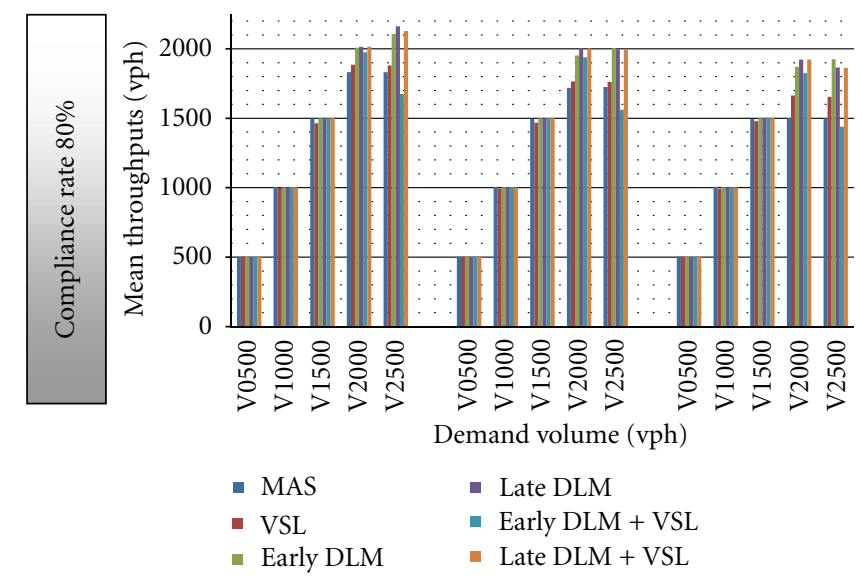

(c)

Figure 4: Mean throughputs.

traffic demand volume levels are created. V0500 means the traffic demand volume is $500 \mathrm{veh} / \mathrm{hr}$. V1000 means the traffic demand volume is $1000 \mathrm{veh} / \mathrm{hr}$. V1500 means the traffic demand volume is $1500 \mathrm{veh} / \mathrm{hr}$. V2000 means the traffic demand volume is $2000 \mathrm{veh} / \mathrm{hr}$. V2500 means the traffic demand volume is $2500 \mathrm{veh} / \mathrm{hr}$. For each run or combination, 10 iterations with different seed number were completed.

5.1. Work Zone Throughputs. The objective of this simulation study is to determine the MOT with the best performance under different combinations. Figure 4 shows the average throughputs under each combination mentioned above. Ideally, one would like to know under each demand volume level, each compliance rate level, and truck percentage level, which MOT type results in the highest throughout. Therefore, for each combination pair wise Tukey's comparisons were completed to determine whether there is a significant difference between throughput means. In Table 1, "blank cells" mean that there was no statistically significant difference between throughput means. The highlighted cells indicate a statistical difference at 0.05 significance level. The same table shows number ranging from 1 to 6 . One is the best case with the highest throughput mean and 6 is the lowest throughput mean. From Table 1, and as shown in Figure 4, one can notice that under demand volumes V500, V1000, and V1500, there were no significant differences in the mean throughputs for all compliance rates and trucks percentage in the traffic composition. However, there are differences in the mean throughputs for the demand volume levels of V2000 and V2500.

From Figure 4 and Table 1, under demand volumes of V2000 and V2500, mean throughputs generally increase as the compliance rate increases for the late DLM, late DLM combined with VSL, and early DLM MOTs. As for VSL only and early DLMs combined with VSL MOTs, Figure 4 and Table 1 show that the resulting mean throughput is significantly lower than the mean throughputs for other MOTs. For example, for compliance rate of $80 \%$, and for all truck percentages in the traffic composition, one can see that the mean throughput of the early DLM combined with VSL MOT drops significantly compared to the same MOT under compliance rate of $20 \%$ or $40 \%$ and at high demand volumes of 2,500 veh/hr. From Table 1 and Figure 4, under higher demand volume levels (V2000 and V2500), late DLM with and without VSL produced higher mean throughputs for all compliance rates and truck percentages.

Results show that among all MOT plans, the MAS and VSL only scenarios resulted in the lowest/worst mean throughputs under all demand volume levels, percentage of trucks in the traffic composition, and compliance rates. 


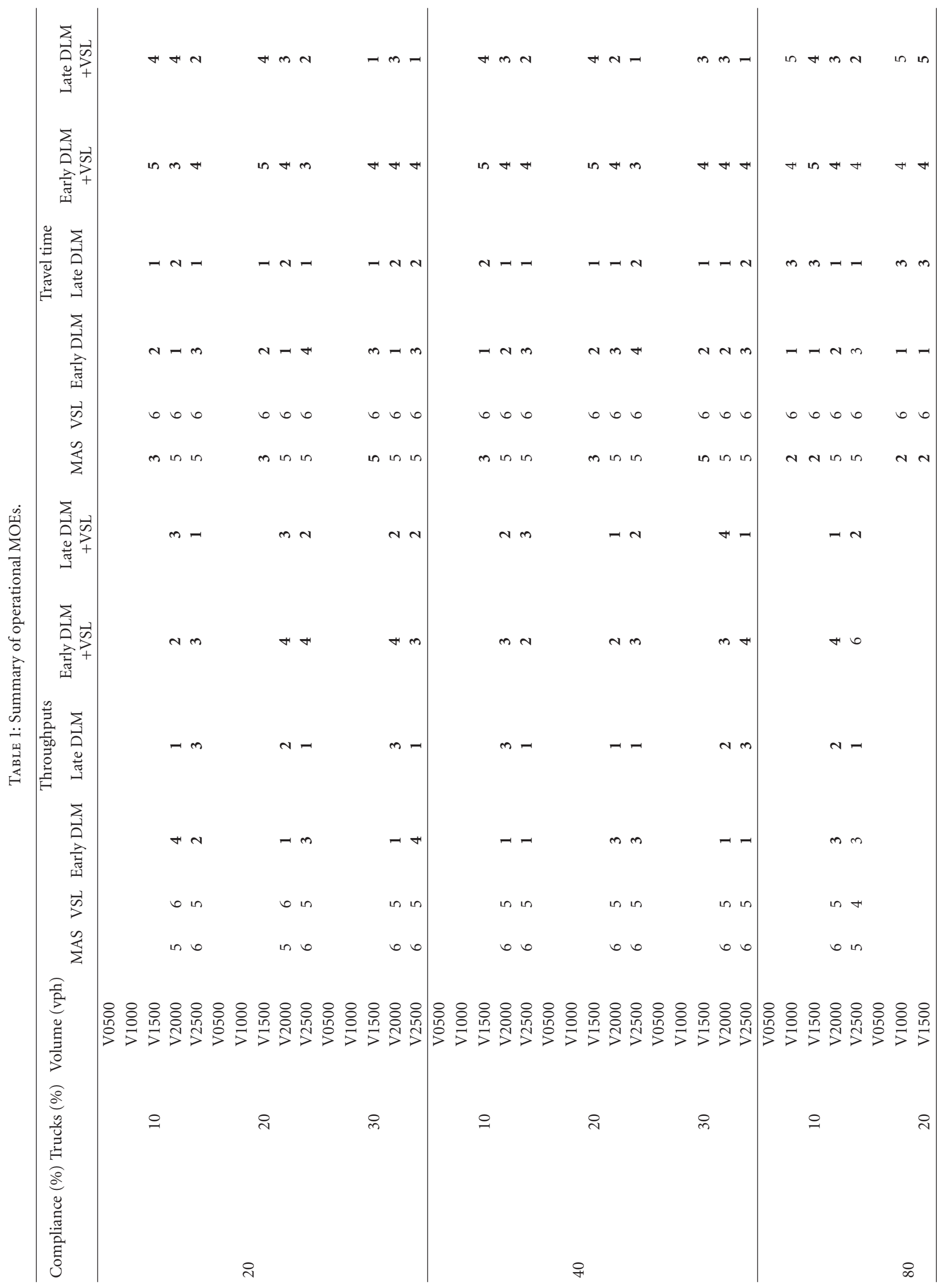




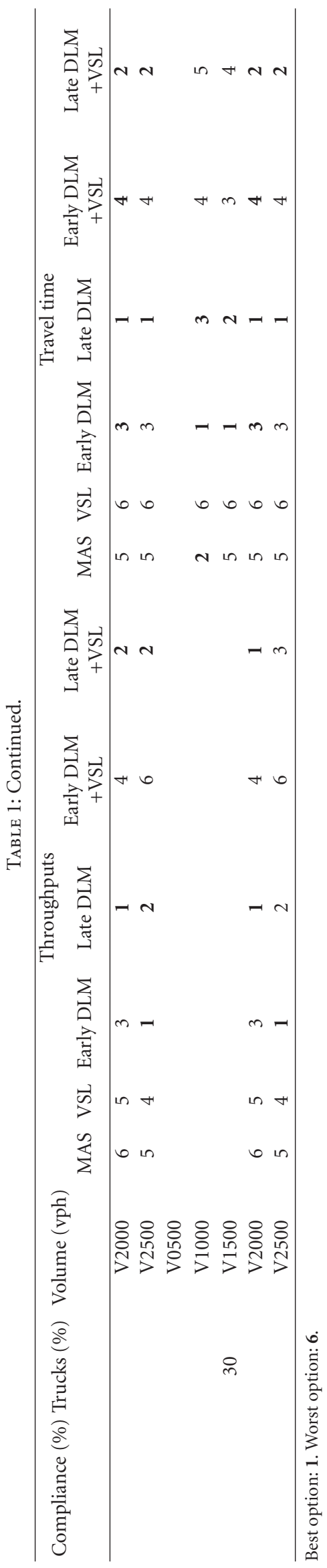




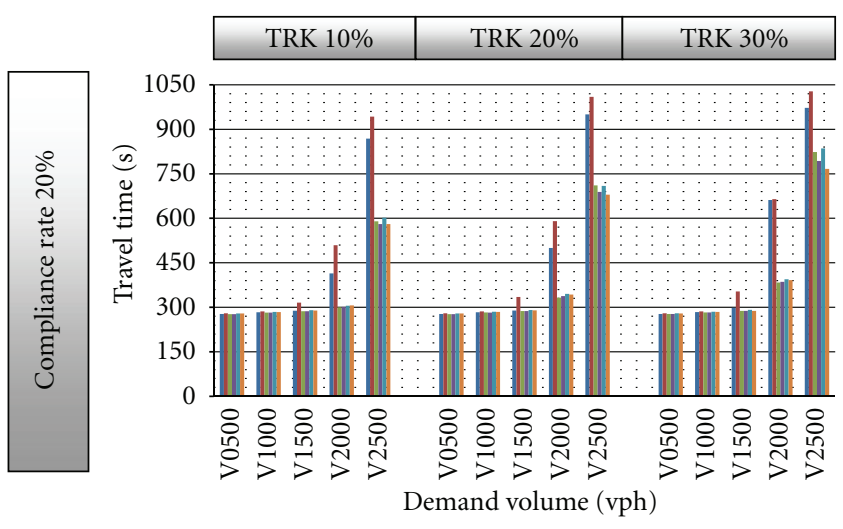

(a)
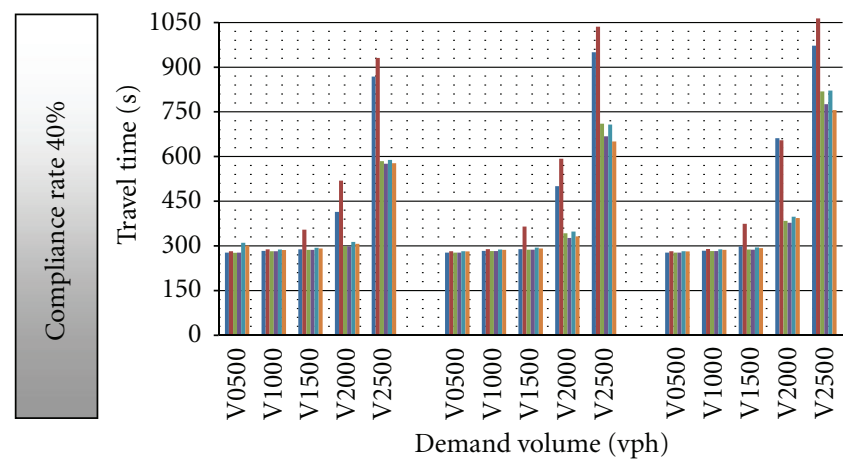

(b)

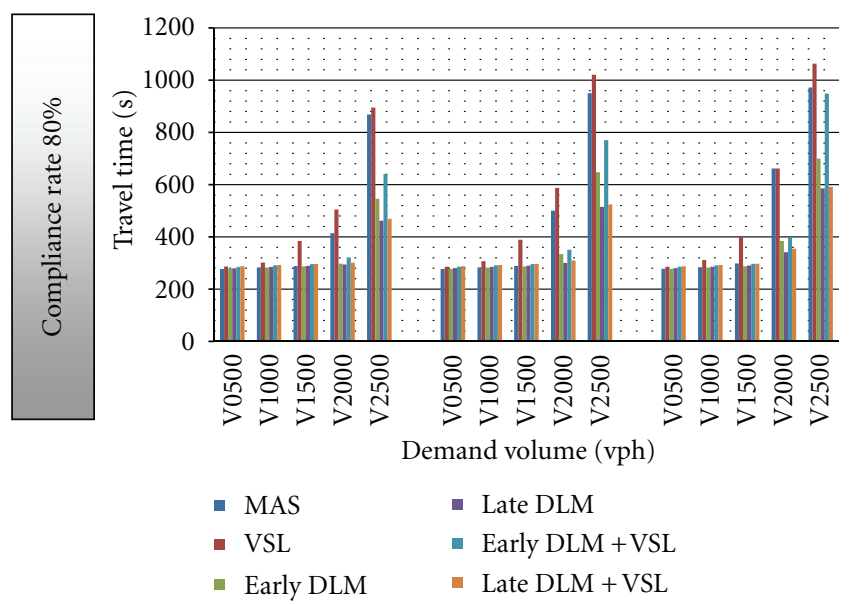

(c)

FIGURE 5: Mean travel times.

5.2. Travel Time through the Work Zone. Figure 5 shows the average travel times under each combination of demand volume, truck percentage in the traffic composition, compliance rate, and MOT type. For each combination pair wise Tukey's comparisons were completed to determine the difference between each pair of throughput means. In Table 1, "blank cells" mean that there was no statistically significant difference between travel time means. Highlighted cells mean that there was a significant difference amongst travel time means.

Results showed that under demand volume levels of V0500 and V1000, different MOT types did not result in statistically different travel time means (see Figure 5 and Table 1). Results also indicated that for demand volume of V1500, the travel time for VSL is the worst as compared to other MOT types. Results showed that compliance rates and truck percentages affect travel time significantly. Table 1 and Figure 5 show that under demand volumes of V0500, V1000, and V1500, travel time for all the MOT types increases with the increase in compliance rate for any given percentage of trucks.

For high demand volume of 2,000 vph, early DLM, late DLM, and late SDLM combined with VSL showing improvement in reducing travel times as the compliance increased compared to the standard MOT using by the state of Florida. In fact, VSL alone and MAS result in the highest travel times for all compliance rates and truck percentages for demand volumes V2000 and V2500.

From Table 1, results show that at high compliance rates (80\%), the early DLM resulted in the best travel times under demand volumes of $1,000 \mathrm{veh} / \mathrm{hr}$ and $1,500 \mathrm{veh} / \mathrm{hr}$ for all truck percentages in the traffic composition. Under demand volumes of 2,000 veh/hr and 2,500 veh/hr, for all truck percentages in the traffic composition and at $80 \%$ compliance rates, the late DLM resulted in the best travel times. Under lower compliance rate levels (20\% and 40\%) the early DLM and late DLM resulted in the best travel times for all demand volume levels.

\section{Conclusions and Recommendations}

A real work zone with a two-to-one lane closure configuration was replicated in VISSIM and calibrated and validated in an earlier study. This study extends the fields study by simulating the same work zone under different ITS technologies. Six MOT types were simulated:

(i) work zone without VSL and without DLM,

(ii) work zone with VSL and without DLM, 
(iii) work zone with VSL and Early DLM,

(iv) work zone with VSL and Late DLM,

(v) work zone with early DLM and without VSL,

(vi) work zone with late DLM and without VSL.

Travel times and work zone throughputs were the selected measure of effectiveness. Table 1 , summarizes the results of this study. In fact, for each combination the results were numbered 1 , through 6,1 being the best and 6 being the worst. The cells left blank in the table reflect no significant difference between the combinations. From Table 1, one can see that in terms of throughputs, there is no difference in all MOT types for low and medium volume levels, that is, V0500, V1000, and V1500. For higher demand volumes (2,000 and 2,500 vph), throughputs for early DLM, late DLM, combination of early DLM with VSL, and combination of late DLM with VSL were significantly larger than throughputs for MAS and VSL alone for all compliance rates and truck percentages. When compliance rates increase under high demand volumes, the combination of VSL and early DLM results in lower throughputs compared to the same MOTs under lower demand volume or compliance rate and compared to other MOTs under the same conditions.

The addition of VSL to the DLM did not significantly improve the work zone throughputs and travel times when compared to DLM without VSL. Since VSLs are primarily added to decrease speed variance and fluctuations in work zones, one may examine and compare the safety aspects of adding a VSL to DLM systems to determine whether this integrated system improves safety of work zones.

Based on the results of this simulation study, one can see that the MAS and VSL MOTs resulted in the lowest operational performance under all demand volume levels, truck percentages in the traffic composition, and compliance rates.

\section{References}

[1] A. Tarko and S. Venugopal, "Safety and capacity evaluation of the Indiana lane merge system," FHWA/IN/JTRP/-2000/19. Purdue University, West Lafayette, Ind, USA, 2001.

[2] A. G. Beacher, M. D. Fontaine, and N. J. Garber, "Evaluation of the late merge work zone traffic control strategy," Tech. Rep. VTRC-05-R6, Virginia Transportation Council, 2004.

[3] A. Tarko, S. Kanipakapatman, and J. Wasson, "Modeling and optimization of the Indiana Lane merge control system on approaches to freeway work zones," Final Report FHWA/ IN/JTRP-97/12, Purdue University, West Lafayette, Ind, USA, 1998.

[4] P. T. McCoy, G. Pesti, and P. S. Byrd, "Alternative information to alleviate work zone related delays," SPR-PL-1 SPR-PL-1(35) P513, University of Nebraska-Lincoln, 1999.

[5] "Dynamic Lane Merge Traffic Control System: A Strategy for Alleviating Aggressive Driving Behavior at Work Zones in Michigan," Department of Civil and Environmental Engineering, Wayne State University, 2001.

[6] P. T. McCoy and G. Pesti, "Dynamic late merge control concept for work zones on rural interstate highway," in Proceedings of the 80th Annual Meeting of the Transportation Research Board, Washington, DC, USA, 2001.
[7] Meyer, E. Construction Area Late Merge (CALM) System, "Midwest Smart Work Zone Deployment Initiative," 2004.

[8] "An Applied Technology and Traffic Analysis Program," University of Maryland, 2006.

[9] URS, "Dynamic Late Merge System Evaluation: Initial Deployment on US-10," Minnesota Department of Transportation, 2003.

[10] URS, "Evaluation of 2004 Dynamic Late Merge System," Minnesota Department of Transportation, 2004.

[11] L. F. Grillo, T. K. Datta, and C. Hartner, "Evaluation of Dynamic late lane merge system at freeway construction zones," in Proceedings of the 87th Annual Meeting of the Transportation Research Board, Washington, DC, USA, 2008.

[12] K. P. Kang, G. L. Chang, N. Zou, and Transportation Research Record, "An optimal dynamic speed limit control for highway work-zone operations," in Proceedings of the Transportation Research Board Annual Meeting Proceedings, 2004.

[13] R. W. Lyles, W. C. Taylor, and D. Lavansiri, "A field test and evaluation of variable speed limits in work zones," in Proceedings of the Transportation Research Board Annual Meeting Proceedings, 2004.

[14] G. Pesti and P. T. McCoy, "Long-Term Effectiveness of Speed Monitoring Displays in Work Zones on Rural Interstate Highways," Transportation Research Record 1754. Transportation Research Board. Washington, DC, USA, 2001.

[15] Florida Department of Transportation, "Plans Preparation Manual (Chapter 10. Work Zone Traffic Control)," 2008.

[16] VISSIM User Manual Version 4.30. P.T.V AG, Karlsruhe, Germany, 2007.

[17] R. Harb, E. Radwan, and M. Abdel-Aty, "Two simplified ITSbased lane management strategies for short term work zones," Journal of Intelligent Transportation Systems, vol. 15, no. 1, pp. 52-61, 2011. 

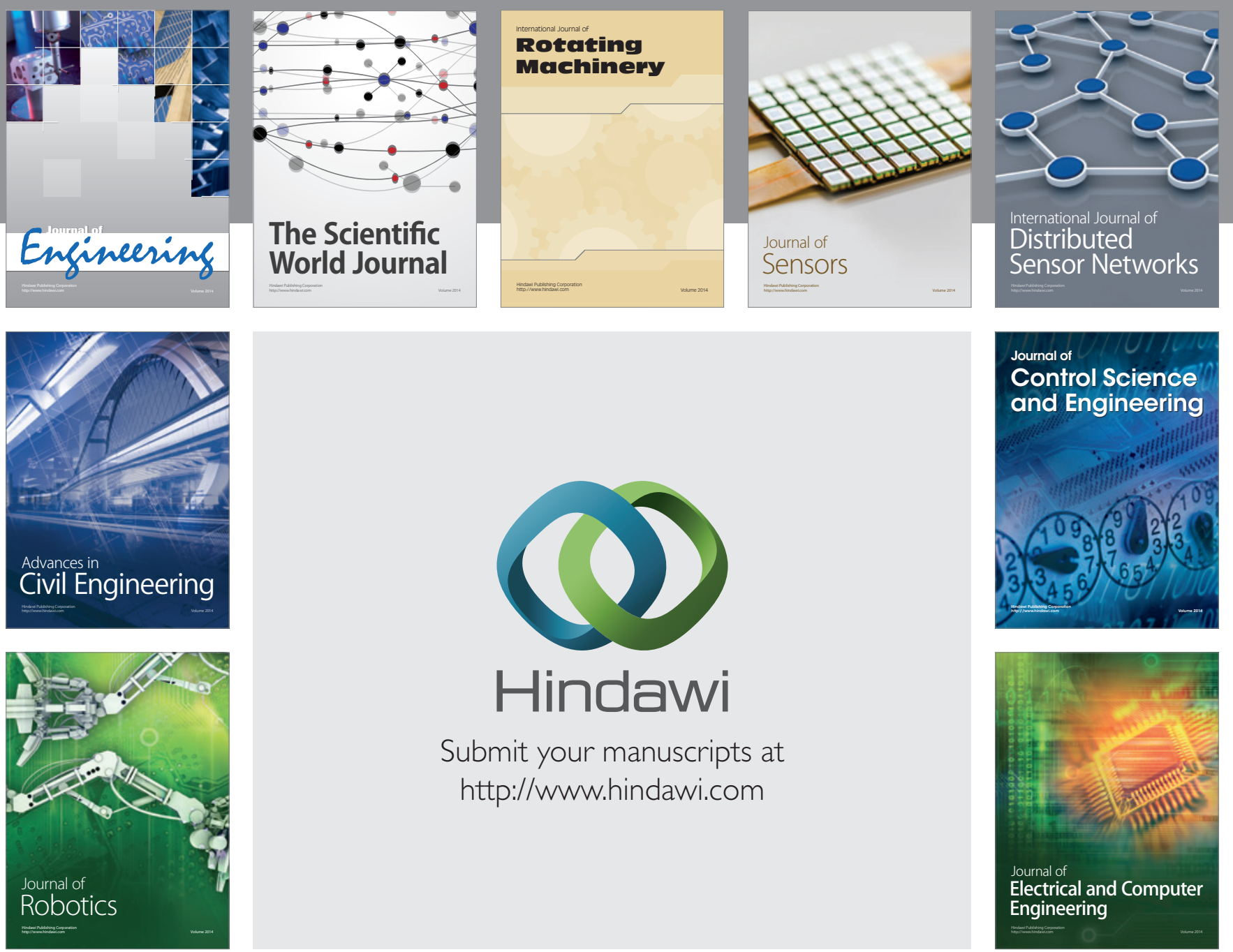

Submit your manuscripts at

http://www.hindawi.com
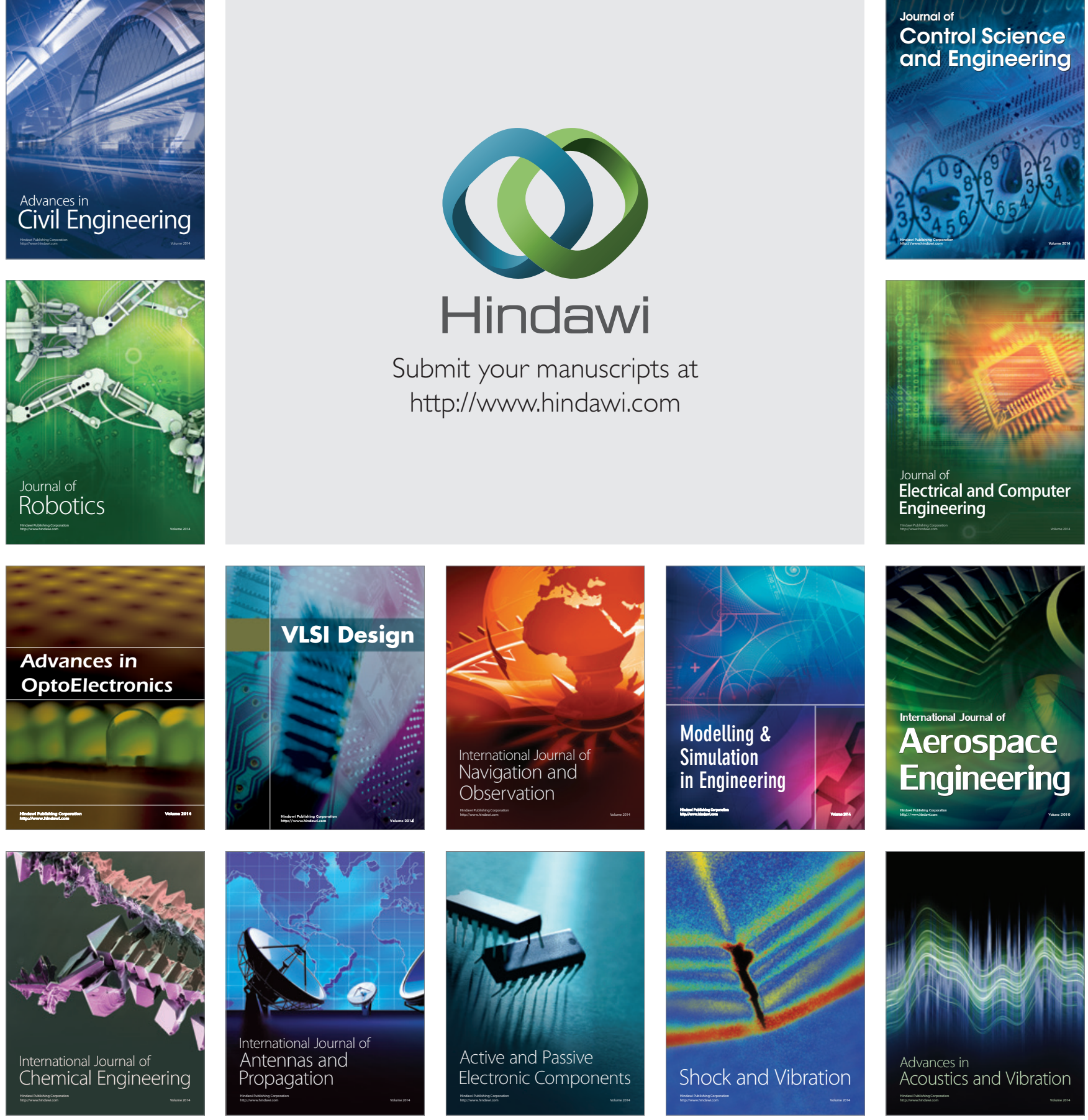